\title{
Body Focused Repetitive Behaviour - Inclusion of Trichotillomania and Other Related Disorders : Recent Update
}

\author{
Seshadri Sekhar Chatterjee, Didakamiwan Khonglah
}

\section{INTRODUCTION}

The term body-focused repetitive behaviours (BFRBs) refers to a group of problematic, destructive, and apparently non-functional behaviours directed toward the body, including hair-pulling (trichotillomania; TTM), skin-picking, and nailbiting $^{1}$. Nosologically, it is proposed in upcoming ICD 11 classification and currently mentioned under Obsessive Compulsive related disorder in the ICD 11 Beta draft and DSM 5. Though being two unrelated disorder, as they were considered previously, these disorders always creates a diagnostic challenge to clinicians over the last few years due to their diagnostic instability, unreliability, phenomonological impasses and lack of common heuristic explanations.

\section{CONCEPT AND NOSOLOGY OF BFRB}

The term body-focused repetitive behaviours (BFRBs) refers to a group of problematic, destructive, and apparently non-functional behaviours directed toward the body, including hair-pulling (trichotillomania), skin-picking, and nail-biting ${ }^{2}$. Over the years there has been no clear method of their classification. Many people fail to realize that it is a mental condition. Clinicians on the other hand have a hard time whether to group them as habits, impulse control problems or compulsions. DSM V has clubbed this under Obsessive-compulsive and related disorders as this is how most clinicians see these behaviours ${ }^{2}$. The term Body focused repetitive behaviour was suggested by Bohne et al (2002) and body focussed repetitive disorder is recommended by ICD 11 to promote global identification and treatment for these conditions. A reason to consider while grouping these conditions together is the fact that they are all directed towards one's own body. A reason for concern is that repetitive behaviours can lead to serious physical and social consequences. Research into Body focused related behaviours is limited as compared to other mental disorders.

\section{ETIOLOGY}

Little is known about the causes of BFRB but various models have been presented like :

1. Psychodynamic model - It is a representation of unresolved conflicts or as a result of disrupted psychosexual development. It also represents an effort to cope with real or threatened object loss.

2. Cognitive behavioural model - This model adds that maladaptive behaviors are often triggered by distorted or unhelpful thoughts and that maladaptive behavior is maintained by positive or negative reinforcement. CBM includes A. Comprehensive behavioural model It incorporates internal and external triggers for episodes of BFRB, factors that facilitate or inhibit the behavior, and positive and negative reinforcement of the behavior once it has been initiated. B. Emotion regulation model which refers to the ways in which individuals identify (i.e., attend to, label, evaluate) and respond to (i.e., express, try to modify) emotional experiences. C. Stimulus regulation model Individuals with TTM pull out hair both when they are overstimulated (due to stress 
or to positive or negative excitement) and understimulated (due to inactivity or boredom). Individuals with BFRB have different threshold for physiological stimulation.

\section{TRICHOTILLOMANIA}

TTM is characterized by recurrent pulling of one's hair resulting in hair loss. It was first included in DSM III under ICD not classified elsewhere in $1990^{3}$. In ICD-10, trichotillomania is classified in the section on disorders of adult personality and behaviour, as one of the habit and impulse disorders. In ICD 11 beta draft TTM and Skin picking was included under OC or related disorders (BFRB). Modifications from DSM-III-R to DSM-IV included expansion of criterion $\mathrm{B}$ to include tension experienced when attempting to resist hair pulling and the addition of a clinical significance criterion $\mathrm{E}$, which required distress and/or impairment. In DSM V it was classified under OCRD in view of its overlapping features with OCD. DSM-5 requires that hair pulling lead to hair loss, but unlike DSM-IV, the new criterion does not require that the hair loss be "noticeable." DSM-5 has added a new criterion: namely, that the person has made repeated attempts to decrease or stop hair pulling ${ }^{3}$.

It occurs in $10 \%$ of the population with the age of onset before menarche. It is more common in females (9:1). There has been evidence of the involvement of CSTC circuit, deficits in working memory and visuospatial learning. Candidate genes include SAPAP3, SLITRK1. (Lovato 2013).

Clinical features include automatic (less aware of the hair pulling) and focused pulling (aware of an urge to pull, resist it and then gives in). Not all who reports of hair pulling reports of tension prior to pulling and it is not clear whether this tension is generated by external factors or it is generated by efforts to resist the pull.

\section{SKIN PICKING DISORDER:}

Pathological skin-picking (SP) refers to repetitive picking of skin or scabs, causing tissue damage and distress or impairment, in the absence of a dermatological condition. Common sites include accessible areas like the face, upper body, cuticles ${ }^{6}$.

Skin picking was finally recognized as a disorder in DSM-5. Given the growing body of data emphasizing that skin-picking disorder is a prevalent and disabling condition, it was considered appropriate for inclusion in DSM-5 as excoriation (skin picking) disorder in the chapter on OCRD. DSM-5 also requires that the person have tried on several occasions to decrease or stop the picking. DSM-5 included skin-picking disorder as a separate disorder because of evidence for its unique biology and clinical presentation. Similarly ICD 11 beta draft includes it under OC and related disorders (BFRD).

It occurs in $1.4-5.4 \%$ of the population with a mean age of onset of about 12 years and shows involvement of CSTC circuit, genetic involvement of SAPAP3 gene. It is trigerred by hyperarousal (stress) or hypoarousal ${ }^{6}$.

\section{NAIL BITING DISORDER}

It is defined as insertion of the fingers into the mouth with contact of the nails with the teeth. In some severe cases the biting can go up to the nail bed and cuticle. NB is usually considered a habit until it causes significant tissue damage and social distress. The prevalence rate peaks at puberty with the rate ranging from $25 \%-60 \%$. It was not listed in DSM-IV or ICD 10 but included in DSM 5 after the field survey. (Siddiqui, 2017)

\section{PROBLEMS ARISING FROM ICD-10 AND DSM-5}

The first issue for ICD-11 concerns the names for these two disorders. Certain individuals and advocacy groups have suggested that the term 


\section{Body Focused Repetitive Behaviour - Recent Update}

is stigmatizing due to the mania suffix, which suggests a relationship to bipolar disorder ${ }^{7}$. Some publications have suggested traction alopecia, hairpulling disorder, or trichotillia; and whether the diagnostic criteria for trichotillomania are suitable cross-culturally, from a developmental perspective, and for both genders'. Some data suggest that there are differences between focused and automatic hair pulling, and between early-onset and late-onset trichotillomania, the question arises as to whether subtypes should be included in the diagnosis. Further trials are needed on this matter and hopefully ICD 11 will come with a more homogeneous group in this regards.

\section{REFERENCES}

1. Snorrason, I., Belleau, E. L., \& Woods, D.W. (2012). How related are hair pulling disorder (trichotillomania) and skin picking disorder? A review of evidence for comorbidity, similarities and shared etiology. Clinical Psychology Review, 32, 618-629.
2. American Psychiatric Association. Diagnostic and Statistical Manual of Mental Disorders. 5th ed. Arlington, VA : American Psychiatric Publishing; 2013.

3. World Health Organization. International Statistical Classification of Diseases and Related Health Problems. 10th rev. Geneva : WHO; 1992.

4. Lochner C, Grant JE, Odlaug BL, Stein DJ. DSM-5 field survey : skin picking disorder. Ann Clin Psychiatry. 2012; $24: 300-4$

5. Lovato L, Ferrão YA, Stein DJ, Shavitt RG, Fontenelle LF, Vivan A, et al. Skin picking and trichotillomania in adults with obsessive-compulsive disorder. Compr Psychiatry. 2012; $53: 562-8$.

6. Odlaug BL, Grant JE. Clinical characteristics and medical complications of pathologic skin picking. Gen Hosp Psychiatry. 2008; $30: 61-6$.

7. Grant JE, Odlaug BL, Hampshire A, Schreiber LR, Chamberlain SR. White matter abnormalities in skin picking disorder: a diffusion tensor imaging study. Neuropsychopharmacology. 2013; $38:$ 763-9.

8. Siddiqui, J. A., Qureshi, S. F., Marei, W. M., \& Mahfouz, T. A. (2017). Onychophagia (Nail Biting): A Body Focused Repetitive Behavior due to Psychiatric Co-morbidity. Journal of Mood Disorders (JMOOD), 7(1), 47-9.

9. Monzani B, Rijsdijk F, Cherkas L, Harris J, Keuthen N, Mataix-Cols D. Prevalence and heritability of skin picking in an adult community sample : a twin study. Am J Med Genet B Neuropsychiatr Genet. 2012; 159B : 605-10. 